\section{Vergleich zwischen anteriorer Rhinomanometrie und impulsoszillometrischer Rhinometrie bei nasalen Allergenprovokationen ${ }^{1}$}

\begin{abstract}
Zusammenfasssung: Neben dem Standardverfahren der anterioren Rhinomanometrie $(\mathrm{aR})$ steht die impulsoszillometrische Rhinometrie (IOS) als Messmethode des nasalen Widerstandes zur Verfügung. Wir überprüften, ob die Resistancemessung mittels IOS ein geeignetes Verfahren bei Allergenprovokationen darstellt. Methode: Es wurden bei 17 Patienten mit Rhinokonjunktivitis $(6 \mathrm{w}, 11 \mathrm{~m})$ nach vorausgegangenem Pricktest nasale Provokationstestungen mit einem entsprechenden Allergen durchgeführt und dabei die Gesamt-Resistance der Nase in randomisierter Reihenfolge mittels aR und IOS gemessen. Ergebnisse: Die Gesamt-Resistance zeigte weder bei der Leermessung (aR $0,38 \pm 0,14 \mathrm{kPa} / \mathrm{l} / \mathrm{s} ;$ IOS $0,38 \pm 0,11 \mathrm{kPa} / \mathrm{l} / \mathrm{s}$ ), nach Kontrolllösung (aR 0,38 $\pm 0,14$; IOS $0,39 \pm 0,14$ ) noch nach Applikation der Allergenlösung (15 min: aR $0,69 \pm 0,27$; IOS $0,77 \pm 0,42$; 30 min: $a R \quad 0,65 \pm 0,29$; IOS $0,6 \pm 0,38$ ) einen signifikanten Unterschied zwischen den beiden Verfahren. Die Messwerte der aR und IOS nach Allergenlösung wiesen eine positive Korrelation auf (15 min: $r=0,63, p<0,01$; 30 min: $r=0,67$, $p<0,01)$. Bei den Messergebnissen mit positiver Reaktion in der aR zeigte sich in 6 von 7 Fällen eine Übereinstimmung. 4 der 7 Patienten mit negativer Reaktion in der aR wiesen unter Zugrundelegung derselben Kriterien in der IOS einen signifikanten Widerstandsanstieg auf. Schlussfolgerung: IOS stellt ein geeignetes Verfahren bei nasalen Provokationstestungen dar und bietet gegenüber der aR den Vorteil der Unabhängigkeit von der Mitarbeit des Patienten. Wegen der höheren Empfindlichkeit der IOS sind die gültigen Grenzwerte für die aR bei Provokationstestungen zur Vermeidung falsch positiver Resultate nicht ohne weiteres auf diese zu übertragen.
\end{abstract}

Comparison Between Anterior Rhinomanometry and Impulse-oscillometric Rhinometry Found Within Nasal Allergen Provocation: Besides the standard method of anterior rhinomanometry (aR), the impulse-oscillometric rhinometry (IOS) is available for measurements of the nasal resistance. The $a R$ is a procedure dependent on the cooperation of the patient, whereas IOS is measured regardless from the breathing activities of the patient. We examined wheter the resistance-measurement by means of IOS in comparison to the $\mathrm{aR}$ is a more suitable method for nasal allergic provocation. Method: 17 patients with anamnestic known rhinokonjunktivitis $(6 \mathrm{f}, 11 \mathrm{~m})$ had a pricktest and then a nasal provocation testing with an allergen which provoked a reaction on the skin. The complete resistance of the nose was measured in a randomized order by means of $\mathrm{aR}$ and

Pneumologie 2001; 55: 420-424

(c) Georg Thieme Verlag Stuttgart · New York ISSN 0934-8387
W. Galetke, W. Randerath, M. David, K.-H. Rühle

Klinik für Pneumologie, Allergologie und Schlafmedizin Universität Witten/Herdecke Klinik Ambrock, Hagen (Professor Dr. med.K.-H. Rühle)
IOS. Results: The complete resistance showed neither in the basic measurement (aR 0,38 $\pm 0,14 \mathrm{kPa} / \mathrm{l} / \mathrm{s}$; IOS $0,38 \pm 0,11 \mathrm{kPa} / \mathrm{l} / \mathrm{s}$ ) nor in the control solution ( $\mathrm{aR} 0,38 \pm 0,14$; IOS $0,39 \pm 0,14$ ) nor after application of the allergenic solution (15 min: aR $0,69 \pm 0,27$; IOS $0,77 \pm 0,42 ; 30 \mathrm{~min}$ : aR $0,65 \pm 0,29$; IOS $0,6 \pm 0,38$ ) a significant difference between the two methods. The results of the measurement of aR and IOS after the allergenic solution showed a positive correlation ( $15 \mathrm{~min}: r=0,63$, $p<0,01 ; 30$ min: $r=0,67, p<0,01)$. We found by means of clinic and measurement methods in the $\mathrm{aR}$ of 7 patients a positive reaction, within 7 patient a negative reaction, 3 patients had a unspecific nasal hyperreactivity. There was a correspondence in 6 of the 7 patients with positive reaction in aR between both methods. 4 of the 7 results with negative reaction in the aR would have been positive then underlying the same criteria in the IOS without announcing the symptomscore a relevant clinical symptomatic while testing. Conclusion: IOS is a suitable procedure for nasal provocation testing and provides results similar to the $\mathrm{aR}$. In comparison to $\mathrm{aR}$ IOS is not dependent on the patients cooperation. Due to its higher sensitivity the valid limits of the aR at provocation testings cannot be transferred to IOS to avoid a false positive reaction.

\section{Einleitung}

Nasale Provokationstestungen (NPT) dienen dem Nachweis einer klinisch relevanten Sensibilisierung, insbesondere bei Diskrepanzen zwischen Anamnese und Hauttestungen bzw. Antikörpernachweis [1-10]. Beurteilt werden beim NPT im Wesentlichen die Irritation, Sekretion und Obstruktion der Nasenschleimhaut, letztere wird durch Messung des nasalen Strömungswiderstandes objektiviert [11].

Als Goldstandard gilt heute bei der NPT die aktive anteriore Rhinomanometrie (aR) [12]. Hierbei wird der Volumenfluss mittels Pneumotachographen bestimmt und das kontralaterale Nasenostium durch einen Messschlauch verschlossen, über den der Differenzdruck zwischen Naseneingang und Choane gemessen wird. Die Durchführung der aR ist mitarbeitsabhängig, da forcierte Atemmanöver des Patienten zu einem Anstieg der Strömungswiderstände in der Nase u.a. durch die Erzeugung von Turbulenzen führen und auf diesem Wege das Ergebnis einer Provokationstestung beeinflussen können.

\footnotetext{
${ }^{1}$ Der Inhalt der Arbeit wurde auf dem 41. Kongress der Deutschen Gesellschaft für Pneumologie in Hamburg, 1. - 4. 3. 2000, vorgetragen.
} 
Bei der Impulsoszillometrie (IOS), deren Anwendung als nasale Widerstandsmessung mit impulsoszillometrischer Rhinometrie bezeichnet wird, erzeugt ein Lautsprecher oszillierende Druck-Flow-Signale, die der Atmung des Patienten überlagert werden [13-17]. Die Druck-Flow-Relation stellt den Widerstand dar und wird bei verschiedenen Frequenzen des Lautsprechersignals angegeben.

Die impulsoszillometrische Rhinometrie hat sich bei unspezifischen nasalen Provokationstestungen mit Histamin als der aR zumindest gleichwertig erwiesen $[18,19]$. Sie bietet aber den Vorteil der Unabhängigkeit von der Mitarbeit des Patienten. Wir stellten uns daher die Frage, wie sich die IOS bei spezifischen Allergenprovokationen im Vergleich zur aR verhält.

\section{Methoden}

\section{Patienten}

Wir untersuchten 17 Erwachsene (11 Männer, 6 Frauen), die in der Anamnese Beschwerden einer saisonalen Rhinokonjunktivitis angaben. Das Alter der Personen betrug 35,9 $\pm 11,2$ Jahre. Alle Untersuchten äußerten schriftlich ihr Einverständnis zur Teilnahme an der Studie.

\section{Als Ausschlusskriterien galten:}

Schwangerschaft, akut entzündliche Erkrankung der Nase oder Nasennebenhöhlen in den letzten vier Wochen, Operation oder Verletzung der Nase in den letzten drei Monaten, Nachweis einer aktuellen obstruktiven Ventilationsstörung $\left(\mathrm{FEV}_{1}<75 \%\right.$ Soll vor Testung), Medikation mit Cromoglicinsäure nasal in den letzten drei Tagen, mit nasalen Kortikoiden in den letzten 14 Tagen, mit Antihistaminika in den letzten 7 Tagen, systemische Steroide $>10 \mathrm{mg} / \mathrm{d}$ Prednisolon zum Zeitpunkt der Untersuchung.

\section{Testungen}

Es erfolgte zunächst eine Pricktestung mit häufigen ubiquitären Allergenen. Die nasale Provokationstestung wurde dann mit einem Allergen durchgeführt, das im Pricktest eine mindestens histaminäquivalente Reaktion gezeigt hatte. Vor Durchführung der Provokationstestung erfolgte eine Spirometrie und Ganzkörperplethysmographie. Nach Messung der Ausgangswerte wurden die Kontrolllösung auf die untere Nasenmuschel appliziert und die Widerstände beider Nasengänge bestimmt. Anschließend wurde die Allergenlösung in identischer Weise eingesprüht. 15 bzw. 30 Minuten nach Applikation der Allergenlösung erfolgte die Messung der Nasenwiderstände in randomisierter Reihenfolge mittels anteriorer Rhinomanometrie und impulsoszillometrischer Rhinometrie. Es wurden mit beiden Verfahren seitengetrennt die Nasenwiderstände ermittelt und anschließend der Gesamtwiderstand der Nase nach der Formel für parallele Widerstände errechnet:

$$
\frac{1}{R_{\text {gesamt }}}=\frac{1}{R_{\text {rechts }}}+\frac{1}{R_{\text {links }}}
$$

Gemäß den Richtlinien für die Durchführung von nasalen Provokationstests wurde die klinische Symptomatik 15 bzw. 30 Minuten nach Allergenapplikation anhand eines Scores notiert und eine Reaktion in der anterioren Rhinomanometrie als positiv beurteilt, wenn der Symptomen-Score mehr als 3 Punkte erreichte oder ein Anstieg des Widerstandes nach Allergengabe um mehr als 60\% verglichen mit dem Wert nach Lösungsmittel gemessen wurde. Als unspezifische Reaktion wurde ein Widerstandsanstieg um mehr als $30 \%$ bereits nach Gabe des Lösungsmittels gewertet.

\section{Geräte und Materialien}

Zur anterioren Rhinomanometrie diente das Gerät Rhinoscreen ${ }^{\circledR}$ (Fa. Jaeger, Hochberg), wobei die Messung mittels Nasenoliven erfolgte. Das Gerät IOS-Rhino ${ }^{\circledR}$ (Fa. Jaeger) wurde zur Messung der impulsoszillometrischen Rhinometrie genutzt. Hier wurde als Adaptation eine Gesichtsmaske angewandt. Als Substanzen wurden $\mathrm{NaCl} 0,9 \%$, Phenol 0,4\% und kommerzielle Allergenlösungen (Fa. Allergopharma, Reinbek) benutzt. Die Applikation der Substanzen erfolgte über Zerstäuber (Fa. Allergopharma), wobei je 2 Hübe mit jeweils $0,05 \pm 0,005 \mathrm{ml}$ auf die untere Nasenmuschel gesprüht wurden.

\section{Statistik}

Als statistische Methode zur Prüfung der individuellen Differenzen wurde der t-Test für gepaarte Stichproben eingesetzt. Der Produkt-Moment-Korrelationskoeffizient nach Pearson wurde als Maß für den Zusammenhang verwendet. Die Auswertung erfolgte mit dem Programmpaket SPSS 10.

\section{Ergebnisse}

Die Gesamt-Resistance der Nase zeigte weder bei der Leermessung (aR 0,37 $\pm 0,13 \mathrm{kPa} / \mathrm{ls}$; IOS 0,38 $\pm 0,11 \mathrm{kPa} / \mathrm{l} / \mathrm{s}$ ) noch bei der Messung nach der Kontrolllösung (aR 0,38 $\pm 0,14$; IOS $0,39 \pm 0,14)$ signifikante Unterschiede zwischen aR und der IOS. Nach Applikation der Allergenlösung kam es bei den Verfahren zu einer Erhöhung der Resistance; ein signifikanter Unterschied zwischen der aR und der IOS ließ sich jedoch nach 15 Minuten $(a R 0,69 \pm 0,27$; IOS $0,77 \pm 0,42)$ und nach 30 Minuten (aR 0,65 $\pm 0,29$; IOS $0,6 \pm 0,38$ ) nicht nachweisen. Eine Testperson reagierte 30 Minuten nach der Allergenlösung mit einem vollständigen, nicht mehr messbaren Verschluss der Nasengänge (Abb.1).

Bei der Beurteilung der Testreaktionen in der aR nach den gültigen Richtlinien (s.o.) ergaben sich bei sieben Patienten eine positive Reaktion, bei weiteren sieben eine negative Reaktion und bei drei Patienten eine unspezifische nasale Hyperreagibilität. Die Personen, deren Testreaktion bei der aR als positiv eingestuft worden waren, wiesen ebenfalls zu keinem der vier Messzeitpunkte signifikante Unterschiede zwischen den beiden Verfahren auf (Leer: aR 0,42 $\pm 0,15$ $\mathrm{kPa} / \mathrm{l} / \mathrm{s}$; IOS $0,43 \pm 0,11 \mathrm{kPa} / \mathrm{l} / \mathrm{s}$; Kontrolle: aR $0,4 \pm 0,11$; IOS $0,39 \pm 0,1 ; 15$ Minuten nach Provokation: aR 0,89 $\pm 0,2$; IOS $0,98 \pm 0,41 ; 30$ Minuten nach Provokation: aR 0,74 $\pm 0,23$; IOS $0,84 \pm 0,47)$.

Auch bei der Betrachtung der Messungen, die in der aR eine negative Reaktion gezeigt hatten, waren zwischen aR und IOS weder bei der Leermessung ( $\mathrm{aR} 0,33 \pm 0,15 \mathrm{kPa} / \mathrm{l} / \mathrm{s}$; IOS $0,38 \pm 0,1 \mathrm{kPa} / \mathrm{l} / \mathrm{s}$ ) bzw. der Kontrollmessung (aR 0,34 $\pm 0,19$; IOS $0,35 \pm 0,11$ ) noch bei den Messungen nach Provokation 


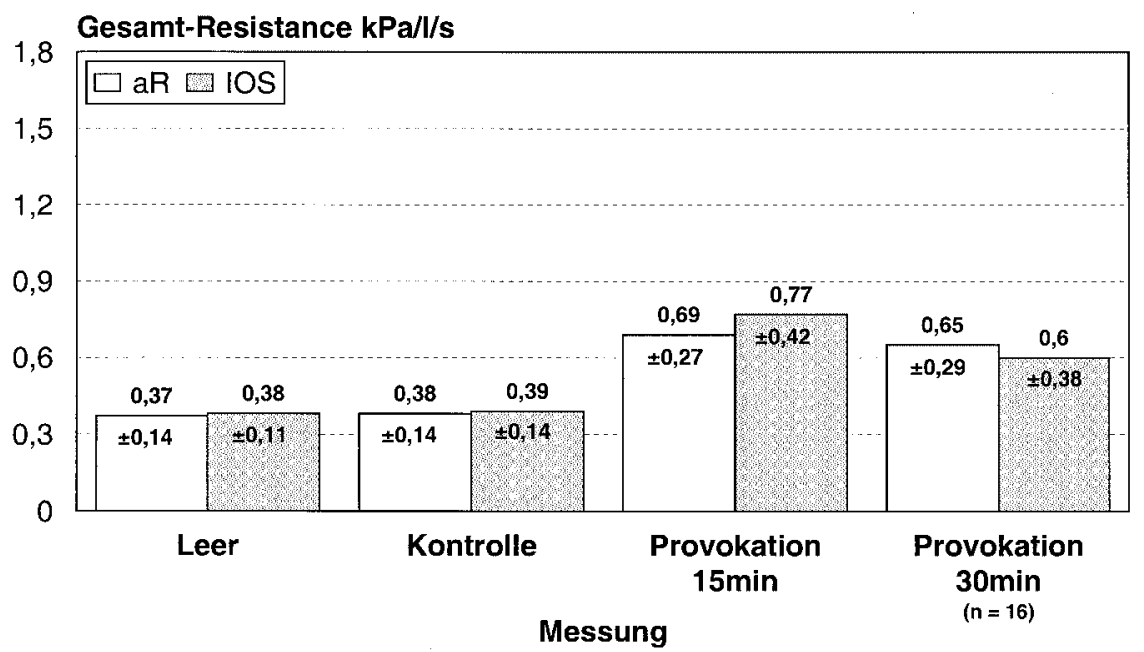

Abb. 1 Mittelwerte und Standardabweichungen der Gesamt-Resistance der Nase beim Vergleich zwischen anteriorer Rhinomanometrie (aR) und impulsoszillometrischer Rhinometrie (IOS) während der Allergenprovokation $(\mathrm{n}=17)$.

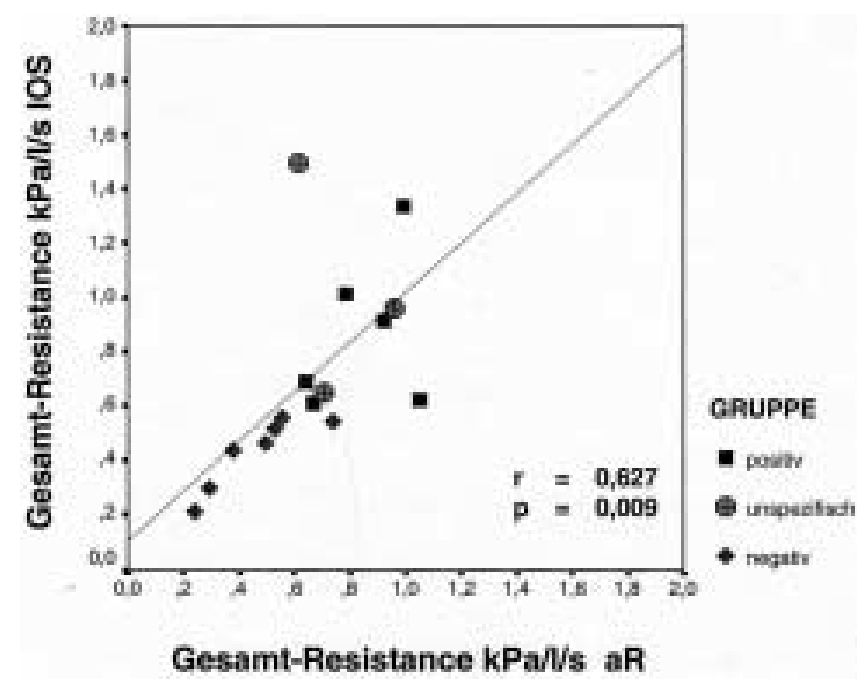

Abb. 2 Korrelation der Gesamt-Resistance der Nase zwischen anteriorer Rhinomanometrie (aR) und impulsoszillometrischer Rhinometrie (IOS) 15 Minuten nach Allergenprovokation.

(15 min: aR 0,46 $\pm 0,17 ;$ IOS $0,43 \pm 0,13 ; 30$ min: aR 0,48 $\pm 0,3$; IOS 0,36 $\pm 0,13$ ) signifikante Unterschiede zu sehen.

Es fand sich bei der Gegenüberstellung der Messwerte in der aR bzw. der IOS 15 Minuten nach Provokation eine positive Korrelation mit einem Korrelationskoeffizienten von 0,627, die Signifikanzniveau erreichte $(\mathrm{p}<0,01)$ (Abb. 2). Ebenso zeigte die Verteilung der Messwerte 30 Minuten nach Provokation eine positive Korrelation $(r=0,673)$ mit $p<0,01$.

Wie oben bereits dargelegt, wird nach den gültigen Richtlinien eine Reaktion im NPT als positiv bewertet, wenn der Widerstandsanstieg nach Allergengabe mehr als 60\% verglichen mit dem Wert nach Lösungsmittel beträgt. Unter Zugrundelegung dieser Kriterien wären bei den Personen, die eine positive Reaktion in der aR zeigten, auch alle Testungen in der IOS als positiv bewertet worden. Lediglich eine Testreaktion (Nr. 2) wäre aufgrund des Widerstandsanstieges auf 147\% nach Applikation der Kontrolllösung als unspezifische Hyperreagibilität gedeutet worden. Der Symptomscore er- reichte mindestens zwei Punkte bei den Messungen, alle Personen wiesen einen klinischen Bezug zu dem getesteten Allergen auf (Tab.1).

Bei den Personen mit einer negativen Testreaktion in der aR wurden bei vier Messungen (Nr. 8, 9,13,14) Widerstandssteigerungen $>60 \%$ nach Allergenapplikation bei der IOS gemessen; diese Reaktionen wären somit als positiv bewertet worden. Zwei Personen (Nr. 13, 14) gaben anamnestisch Beschwerden bei Exposition gegenüber dem getesteten Allergen an, zwei Personen (Nr. 8, 9) verneinten dies. Die klinische Symptomatik war bei den vier Testungen mit 0 bzw. 1 Punkt zu vernachlässigen. Eine Person (Nr.11) wies zudem einen Anstieg des Widerstandes um 44\% nach Kontrolllösung auf und wäre als unspezifisch hyperreagibel eingestuft worden (Tab.1).

Vergleicht man somit die mittels IOS erhobenen Daten mit dem „Goldstandard“ $\mathrm{aR}$, so lägen unter Zugrundelegung derselben Kriterien die Sensitivität der IOS bei $86 \%$ und die Spezifität bei $43 \%$.

\section{Diskussion}

Die Bedeutung der nasalen Provokationstestung zur Objektivierung oder zum Ausschluss einer allergischen Rhinitis liegt darin begründet, dass andere Methoden der Allergiediagnostik wie Anamnese, Hauttestungen oder Bestimmung der spezifischen Antikörper oft keine übereinstimmenden Ergebnisse zeigen $[1,6]$. Insbesondere der Pricktest weist falschpositive Reaktionen in $25-40 \%$ der Fälle auf [6]. Bei nasalen Provokationstestungen im Rahmen arbeitsmedizinischer Fragestellungen spielt die fehlende Abhängigkeit von der Mitarbeit des Patienten eine entscheidende Rolle. Hier weist die als Standardmethode geltende aktive anteriore Rhinomanometrie (aR) jedoch einige Schwächen auf $[8,18,20,21]$, so dass andere Verfahren erprobt wurden. Die impulsoszillometrische Rhinometrie (IOS) zeigte als mitarbeitsunabhängiges Verfahren in früheren Studien bei der Bestimmung des nasalen Widerstandes in Ruhe ähnliche Ergebnisse wie die posteriore Rhinomanometrie [21,22], die passive anteriore Rhinomanometrie [23] und die aktive anteriore Rhinomanometrie $[21,23,24]$. Randerath et al. verglichen die Impulsoszillometrie mit der aR bei nasaler Provokation mit Histamin und 


\begin{tabular}{|c|c|c|c|c|c|c|c|c|}
\hline & Proz & ale Än & ung $n$ & & & & & \\
\hline & Kont & sung & Aller & $15 \mathrm{~min}$ & Allerg & $30 \mathrm{~min}$ & & \\
\hline & $\mathrm{aR}$ & IOS & $\mathrm{aR}$ & IOS & $\mathrm{aR}$ & IOS & $\begin{array}{l}\text { Symptom- } \\
\text { score }\end{array}$ & $\begin{array}{l}\text { Anam- } \\
\text { nese }\end{array}$ \\
\hline 1 & 11 & 25 & 76 & 114 & 90 & 36 & 4 & + \\
\hline 2 & -13 & 147 & 268 & 135 & 1254 & 253 & 3 & + \\
\hline 3 & 19 & 27 & 154 & 164 & 181 & 174 & 3 & + \\
\hline 4 & 20 & 4 & 133 & 92 & 196 & 83 & 3 & + \\
\hline 5 & -20 & -44 & 254 & 245 & 49 & 69 & 3 & + \\
\hline 6 & -47 & -39 & 96 & 128 & 46 & 97 & 2 & + \\
\hline 7 & -5 & -34 & 79 & 47 & 94 & 103 & 2 & + \\
\hline 8 & -20 & 20 & -5 & 103 & -16 & 129 & 1 & - \\
\hline 9 & -3 & 21 & 27 & 5 & 28 & 61 & 1 & - \\
\hline 10 & -8 & 10 & 12 & -3 & -4 & 30 & 4 & + \\
\hline 11 & 7 & 44 & -7 & 17 & -50 & 7 & 2 & - \\
\hline 12 & -36 & 7 & 38 & 19 & 33 & 7 & 0 & - \\
\hline 13 & 12 & -43 & 43 & 135 & 11 & 43 & 0 & + \\
\hline 14 & -1 & -25 & 59 & 86 & 26 & 52 & 1 & + \\
\hline
\end{tabular}

Tab. 1 Prozentuale Änderung der GesamtResistance der Nase bei anteriorer Rhinomanometrie $(\mathrm{aR})$ und impulsoszillometrischer Rhinometrie (IOS) bei Patienten mit positiver Reaktion (Nr. 1-7) bzw. negativer Reaktion (Nr. 8-14) in der aR, Angabe des Symptomscores und des Anamnesebezuges des getesteten Allergens konnten feststellen, dass die IOS der aR zumindest gleichwertig ist [18]. Als geeigneter Parameter fand sich die Resistance bei $5 \mathrm{~Hz}$, so dass wir diese in unserer Studie ebenfalls verwendeten.

Wir konnten bei unseren Messungen nach Applikation der Allergenlösung keine signifikanten Unterschiede hinsichtlich des nasalen Gesamtwiderstandes zwischen IOS und aR feststellen. Dies deckt sich mit einer Untersuchung von Becker et al., die ebenfalls die IOS mit der aR bei nasaler Allergenprovokation verglichen, dabei jedoch nur einseitig die nasale Resistance bestimmten [16].

Frühere Arbeiten über die Existenz sog. nasaler Zyklen wiesen jedoch eine erhebliche Schwankung der Strömungswiderstände in den einzelnen Nasengängen nach, wobei der Gesamtwiderstand weitgehend konstant blieb [25-28]. Aus diesem Grund bestimmten wir bei unserer Untersuchung seitengetrennt die Resistance jedes Nasenostiums und errechneten hieraus die Gesamtresistance der Nase entsprechend dem Modell parallelgeschalteter Widerstände [29].

Es zeigte sich in unserer Studie eine signifikante positive Korrelation zwischen den mit beiden Methoden bestimmten Widerstandswerten. Bei einer früheren Vergleichsstudie zwischen IOS und posteriorer Rhinomanometrie fand sich eine noch größere Übereinstimmung [22]; Petro et al. kamen 1989 jedoch zu ganz ähnlichen Korrelationskoeffizienten beim Vergleich zwischen Rhinomanometer und Oszillationsverfahren (Custovit) [30].

Wir beurteilten in unserer Untersuchung in einem weiteren Schritt zunächst alle Ergebnisse in der $\mathrm{aR}$ nach den gültigen Kriterien und konnten 7 positive Testreaktionen von 7 Patienten mit negativer Reaktion und 3 mit unspezifischer Hyperreagibilität differenzieren. Bei der Betrachtung der mit IOS gemessenen Widerstandswerte bei den Patienten mit einer negativen Testreaktion in der aR fiel auf, dass hier vier
Testergebnisse als positiv und eine Reaktion als unspezifische Hyperreagibilität interpretiert worden wären, d.h. es wären aufgrund der mit der IOS gemessenen höheren Differenz in den Widerstandswerten nach Allergenapplikation immerhin 5 von 7 Testergebnissen anders bewertet worden. Dies deckt sich mit Beobachtungen in früheren Studien, die ebenfalls eine höhere Empfindlichkeit der IOS im Vergleich mit anderen Methoden dokumentierten. So konnten Randerath et al. bei der Histaminprovokation um 50\% niedrigere Grenzkonzentrationen für die Verdoppelung der Resistance bei der IOS gegenüber der aR feststellen [18]. Becker et al. fanden bis $\mathrm{zu} 2 \mathrm{fach}$ erhöhte Resistancewerte bei der IOS im Vergleich mit der aR bei Nasalmessungen [15].

Es stellt sich somit die Frage, ob die in unserer Studie festgestellten Differenzen zwischen der aR und der IOS Ausdruck einer größeren Empfindlichkeit der IOS oder falsch positive Reaktionen der impulsoszillometrischen Methode sind. Für eine höhere Empfindlichkeit der IOS gegenüber der aR spricht die Überlegung, dass sich bei der impulsoszillometrischen Messung die Druck-Flow-Relation annähernd proportional zur Widerstandserhöhung in der Nase ohne Beeinflussung durch Atemmanöver des Patienten verändert, während bei der aR der nasale Fluss durch den Patienten selber erzeugt wird und so eine Obstruktion erst später als bei der IOS erfasst werden kann [31]. Dies würde auch die Ergebnisse der Messungen Nr. 13 und 14 (Tab.1) erklären, bei denen sich bereits bei der aR ein - allerdings nach den gültigen Kriterien noch nicht signifikanter - Anstieg des Widerstandes zeigte, der bei der IOS dann Signifikanzniveau erreichte. Als Hinweis auf eine doch klinisch relevante Reaktion ist auch der positive Anamnesebezug bei diesen Testungen zu werten. Möglicherweise stellt die IOS aufgrund ihrer höheren Empfindlichkeit eine Methode dar, mit der sich bereits beginnende, klinisch noch nicht manifeste Allergenreaktionen an der Nase nachweisen lassen. 
Demgegenüber sind die Testungen Nr. 8 und 9 eher als falsch positive Reaktionen in der IOS zu interpretieren, wenn der geringe Symptomscore und der fehlende Anamnesebezug berücksichtigt wird. Offenbar sind die für die aR gültigen Grenzwerte nicht ohne weiteres auf die impulsoszillometrische Methode zu übertragen.

Zusammenfassend folgern wir aus unseren Untersuchungen, dass die impulsoszillometrische Rhinometrie ein geeignetes Verfahren für die Durchführung von nasalen Provokationstestungen darstellt und dabei der aktiven anterioren Rhinomanometrie in einem weiten Bereich vergleichbare Ergebnisse liefert. Sie bietet den Vorteil der Unabhängigkeit von der Mitarbeit des Patienten und ist somit besonders für arbeitsmedizinische Fragestellungen von Bedeutung. Allerdings sind die für die aR gültigen Grenzwerte der Widerstandsänderung unter der Provokation nicht ohne weiteres auf die IOS zu übertragen, sondern müssen aufgrund der höheren Empfindlichkeit der IOS nach oben korrigiert werden. Daher ist zur Detektion möglicher falschpositiver Testergebnisse insbesondere auf eine Übereinstimmung zwischen impulsoszillometrischer Rhinometrie und klinischer Symptomatik bzw. Anamnesebezug zu achten.

\section{Literatur}

${ }^{1}$ Berdel D, Koch U. Die Bedeutung der „Nasalen Provokation“ in der Allergiediagnostik und ihre Objektivierung mit der Rhinomanometrie. Laryng-Rhinol 1981; 60: 491 - 495

2 Berdel D, Koch U. Messung der Nasenwegswiderstände (Rhinomanometrie) mit der Oszillationsmethode. Laryng-Rhinol 1980; 59: $575-580$

${ }^{3}$ Clement P. Rhinomanometry. Allergy 1997; 52 Suppl 33: 26-27

${ }^{4}$ Fernandez FR, Sole D, Naspitz C, Munoz-Lopez F. Diagnostic value of nasal provocation testing and rhinomanometry in allergic rhinitis. J Invest Clin Immunol 1996; 6 (3): 184-188

${ }^{5}$ Pinkpank A. Beziehungen zwischen Selbsteinstufung der Nasenatmung, Rhinoskopie und Rhinomanometrie. HNO 1986; 34 : $194-197$

${ }^{6}$ Schlenter WW. Die nasale Provokationstestung. Allergologie 1990; 13, 2: $42-52$

${ }^{7}$ Pinkpank A. Messfehler in der Rhinomanometrie. Laryng Rhinol Otol 1986; 65: 256-259

${ }^{8}$ Lund VJ. Objective assessment of nasal obstruction. Otolaryngol Clin North Am 1989; 22 (2): 279-290

${ }^{9}$ Mlynski G, Löw J. Die Rhinoresistometrie - eine Weiterentwicklung der Rhinomanometrie. Laryng Rhinol Otol 1993; 72: $608-610$

${ }^{10}$ Roithmann R, Cole P, Chapnik J, Shpirer I, Hoffstein V, Jamel N. Acoustic rhinometry in the evaluation of nasal obstruction. Laryngoscope 1995; 105 (3Pt1): 275-281

${ }^{11}$ Bachert C, Berdel D. Richtlinien für die Durchführung von nasalen Provokationstests mit Allergenen bei Erkrankungen der oberen Luftwege. Allergologie 1990; 13: 53-55

${ }^{12}$ Malm L, Gerth van Wijk R, Bachert C. Guidelines for nasal provocations with aspects on nasal patency, airflow, and airflow resistance. Rhinology 2000; 38: 1 -6

${ }^{13}$ Vogel J, Smith HJ. Einführung in die Grundlagen der ImpulsOszillometrie. In: Vogel J, Walter D, Smith H-J (Hrsg). Workshop Impuls-Oszillometrie, Bad Reichenhall 1995. München: Dustri, 1995: $12-32$

${ }^{14}$ Becher G, Beck E, Vogel J, Smith HJ. Rhinomanometrie auf der Basis der Impulsoszillometrie. In: Vogel J, Walter D, Smith H-J (Hrsg). Workshop Impuls-Oszillometrie, Bad Reichenhall 1995. München: Dustri, 1995: 60-65
${ }^{15}$ Becher G, Beck E, Smith HJ, Vogel J, Drews D. Anwendung der Impulsoszillometrie (IOS) zur Messung des nasalen Widerstandes bei der nasalen Allergenprovokation. Pneumologie 1994; 48: 859

${ }^{16}$ Becher G, Beck E, Smith HJ. Testung des Rhinomanometers Rhinoscreen der Fa. E. Jaeger bei der nasalen Allergenprovokation. Pneumologie 1994; 48: 860

${ }^{17}$ Rühle KH. Oszillatorische Impedanz - Grundlagen und klinische Anwendungsmöglichkeiten. In: Rühle KH (Hrsg). Oszillatorische Impedanz bei schlafbezogenen Atemregulationsstörungen. Stuttgart: Thieme, 1996: 1-5

${ }^{18}$ Randerath W, Smith HJ, Knarr D, Rühle KH. Vergleich der anterioren Rhinomanometrie und Impuls-Oszillometrie bei nasaler Provokation mit Histamin. Pneumologie 1998; 52: 97-103

${ }^{19}$ Phagoo SB, Watson RA, Pride NB. Use of nasal peak flow to assess nasal patency. Allergy 1997; 52 (9): 901 - 908

${ }^{20}$ Büsser E, Schibli RA. Rhinomanometrie. Methodik und Normalwerte. Dtsch Med Wschr 1973; 98: 719-723

${ }^{21}$ Berdel D, Koch U. Vergleich von aktiver Fremdstromrhinomanometrie (Oszillationsmethode) und aktiver Eigenstromrhinomanometrie bei 17 Patienten vor und nach Nasenscheidewandkorrektur. Arch Otorhinolaryngol 1983; 237: 115-124

${ }^{22}$ Lorino AM, Lofaso F, Abi-Nader F, Drogou I, Dahan E, Zerah F, Harf A, Lorino A. Nasal airflow resistance measurement: forced oscillation technique versus posterior rhinomanometry. Eur Respir J 1998; 11: 720-725

${ }^{23}$ Shelton DM, Pertuze J, Gleeson MJ, Thompson J, Denman WT, Goff J, Eiser NM, Pride NB. Comparison of oscillation with three other methods for measuring nasal airways resistance. Respiratory Medicine 1990; 84: 101 - 106

${ }^{24}$ Hagemann H, Bauer PC, Costabel U. Die nasale Provokationstestung bei Patienten mit Milbensensibilisierung: Ein Vergleich zwischen der anterioren Rhinomanometrie, der Oszilloresistometrie und dem Peak Nasal Inspiratory Flowmeter. Pneumologie 1994; 48: 625

${ }^{25}$ Hasegawa M, Kern EB. The Human Nasal Cycle. Mayo Clin Proc 1977; 52: $28-34$

${ }^{26}$ Syabbalo NC, Bundgaard A, Schmidt A, Widdicombe JG. Measurement and regulation of nasal airflow resistance in man. Rhinology 1986; 24: 87-101

${ }^{27}$ Hasegawa M, Kern EB. Variations in nasal resistance in man: a rhinomanometric study of the nasal cycle in 50 human subjects. Rhinology 1978; 16: 19-29

${ }^{28}$ Raschke F. Tagesrhythmen der nasalen Resistance. In: Rühle KH (Hsrg). Oszillatorische Impedanz bei schlafbezogenen Atemregulationsstörungen.. Stuttgart: Thieme, 1996: 19-25

${ }^{29}$ Deitmer T. Moderne Funktionsdiagnostik der Nase und der Nasennebenhöhlen. European Archives of Oto-Rhino-Laryngology 1996; Supl I: 1 - 71

${ }^{30}$ Petro W, Birkenbihl P. Nasale oszillatorische Widerstandsmessung. Pneumologie 1989; 43: 387 - 391

${ }^{31}$ Hoster M, Schlenker E, Raddatz M, Rühle KH. Oszillatorische Impedanz, Ösophagusdruck und Atemfluss bei Stenoseatmung. Ein Modell für die extrathorakale Obstruktion. In: Rühle $\mathrm{KH}$ (Hrsg). Oszillatorische Impedanz bei schlafbezogenen Atemregulationsstörungen. Stuttgart: Thieme, 1996: 13-18

\section{Dr. med. W. Galetke}

Klinik für Pneumologie, Allergologie und Schlafmedizin Universität Witten/Herdecke

Klinik Ambrock

Ambrocker Weg 60

58091 Hagen 